TITLE:

\title{
Shape-related useful properties of nanostructured thin films
}

$\operatorname{AUTHOR}(S)$ :

Suzuki, Motofumi

CITATION:

Suzuki, Motofumi. Shape-related useful properties of nanostructured thin films. AIP Conference Proceedings: NATIONAL PHYSICS CONFERENCE 2014 (PERFIK 2014) 2015, 1657: 030003

ISSUE DATE:

2015-04-25

URL:

http://hdl.handle.net/2433/210551

RIGHT:

(C) 2015 AIP Publishing LLC 


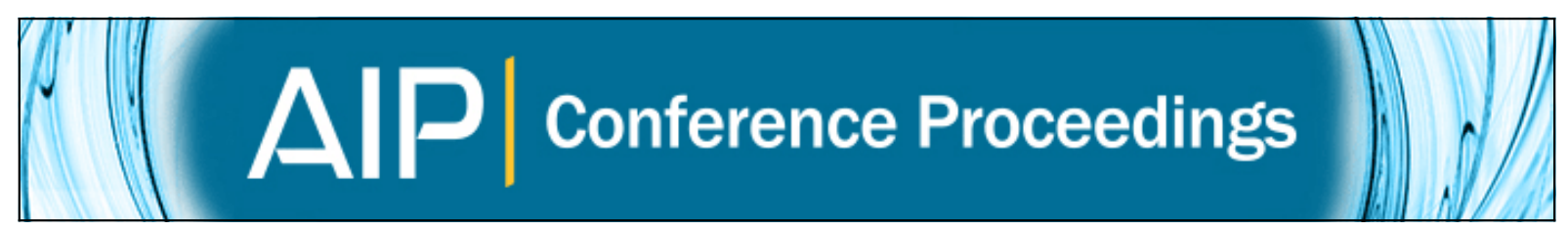

\section{Shape-related useful properties of nanostructured thin films}

Motofumi Suzuki

Citation: AIP Conference Proceedings 1657, 030003 (2015); doi: 10.1063/1.4915153

View online: http://dx.doi.org/10.1063/1.4915153

View Table of Contents: http://scitation.aip.org/content/aip/proceeding/aipcp/1657?ver=pdfcov

Published by the AIP Publishing

\section{Articles you may be interested in}

Comparative study of the mechanical properties of nanostructured thin films on stretchable substrates J. Appl. Phys. 116, 093504 (2014); 10.1063/1.4894616

Optoelectronic Properties of Nanostructured Cadmium Sulphide Thin Films

AIP Conf. Proc. 1391, 585 (2011); 10.1063/1.3643618

Optical, structural, and magnetic properties of cobalt nanostructure thin films

J. Appl. Phys. 105, 113508 (2009); 10.1063/1.3138809

Optical and electrical properties of nanostructured $\mathrm{LaCoO} 3$ thin films

Appl. Phys. Lett. 87, 061909 (2005); 10.1063/1.2009057

Structure-related optical properties of thin films

J. Vac. Sci. Technol. A 4, 418 (1986); 10.1116/1.573894 


\title{
Shape-Related Useful Properties of Nanostructured Thin Films
}

\author{
Motofumi Suzuki
}

Department of Micro Engineering, Kyoto University, Kyoto 615-8540, Japan

\begin{abstract}
The recent status of practical applications of obliquely deposited thin films is reviewed. Owing to the anisotropy in polarizability of elongated nanocolumns, obliquely deposited thin films in the form of assemblies of aligned nanocolumns show anisotropies in various properties, such as birefringence, dichroism, and magnetism. In addition, we introduce examples of practical applications: thin film waveplates, angular selective coatings, Au nanorod arrays for surface-enhanced Raman scattering, and low-reflectivity wire-grid polarizers.
\end{abstract}

Keywords: glancing angle deposition; practical applications; shadowing growth. PACS: $68.55 .-\mathrm{a}, 68.70 .+\mathrm{w}, 78.20 . \mathrm{Fm}, 78.67 . \mathrm{Bf}, 78.67 . \mathrm{Rb}$

\section{INTRODUCTION}

According to elementary electromagnetic theory, dielectric and magnetic polarization phenomena are strongly influenced by the shape of a body, i.e., easy polarization along the elongated side [1,2]. The collective response of well-aligned elongated elements shows various anisotropies in optical [3-5], electrical [6], magnetic [7, 8], and mechanical properties [9] resulting from the shape of the constituent elements. For example, a photonic metamaterial with elongated elements that are much smaller than the optical wavelength exhibits optical anisotropies such as birefringence $[3,4]$ and dichroism $[10,11]$, regardless of the crystallinity of each element. One of the most powerful methods to prepare such metamaterials having shape-related useful properties is the so-called dynamic oblique deposition (DOD) technique $[12,13]$, in which a substrate is set obliquely or sometimes rotated inplane. Through DOD, unique nanocolumnar structures have been prepared by means of the self-shadowing effect and the limited mobility of adatoms. Considerable progress has recently been made in developing products by the DOD technique.

In this presentation, we will discuss the fundamentals of shape-related properties and the DOD technique. We will also introduce our recently commercialized products developed by DOD, namely surface-enhanced Raman substrates [14, 15], thin-film waveplates [16], and low reflectivity wire-grid polarizers [11]. Because DOD films exhibit great potential to overcome the energy and environmental problems confronting humankind, the use of these films in industries should be encouraged by eliminating any negative prejudice against these films.

\section{ORIGIN OF ANISOTOROPY}

Figure 1 shows typical examples of DOD thin films [17]. During conventional PVD, the vapor is deposited on a substrate from the normal direction in vacuum so that flat, uniform thin films are produced. In contrast, during DOD, the substrate is set obliquely and sometimes rotated in-plane, as shown in Fig. 1(g). If the substrate temperature is sufficiently low, unique nanocolumnar structures such as zigzags, helixes, posts, and complex hybrid structures are created [Fig. 1(a)-1(e)]. The physical origins of the columnar structure in DOD thin films are selfshadowing effects and the limited mobility of the deposited atoms. When the vapor flux is obliquely incident on the substrate surface, atoms in the growing films shadow unoccupied sites from the direct sticking of incident atoms ("shadowing effect"). Moreover, owing to limited mobility, the unoccupied sites are not filled later. As a result, oblique columns grow in the direction of the incident vapor beam.

The columnar structures can be modeled as an assembly of elongated nanoellipsoids as shown in Fig. 2(a) [5]. Let us consider polarization properties of each nanoellipsoid (Fig. 2(b)) as expressed by the following equation,

$$
\frac{x^{2}}{a_{1}^{2}}+\frac{y^{2}}{a_{2}^{2}}+\frac{z^{2}}{a_{3}^{2}}=1 \text {. }
$$


When an ellipsoid with dielectric contestant $\varepsilon_{p}$ is embedded in a medium with dielectric constant $\varepsilon_{m}$ and a uniform electric field is applied, the polarizability $\alpha_{i}(i=1,2,3)$ is written as $[1,2]$

$$
\alpha_{i}=4 \pi a_{1} a_{2} a_{3} \frac{\varepsilon_{p}-\varepsilon_{m}}{3 \varepsilon_{m}+3 L_{i}\left(\varepsilon_{p}-\varepsilon_{m}\right)}
$$

where $L_{i}$ is the geometric factor and is written as

$$
\begin{gathered}
L_{i}=\frac{a_{1} a_{2} a_{3}}{2} \int_{0}^{\infty} \frac{d q}{\left(a_{i}^{2}+q\right) f(q)}, \\
f(q)=\sqrt{\left(q+a_{1}^{2}\right)\left(q+a_{2}^{2}\right)\left(q+a_{3}^{2}\right)} .
\end{gathered}
$$

In the case of a needle-like nanospheroid $\left(\alpha_{1} \gg \alpha_{2}=\alpha_{3}\right), L_{1}=0$ and $L_{2}=L_{3}=1 / 2$, so the nanospheroid is easy to polarize along the elongated direction. Therefore, in the form of an assembly of unidirectionally aligned nanospheroids, nanocolumnar thin films show biaxial anisotropy in their optical, electrical, and magnetic properties.

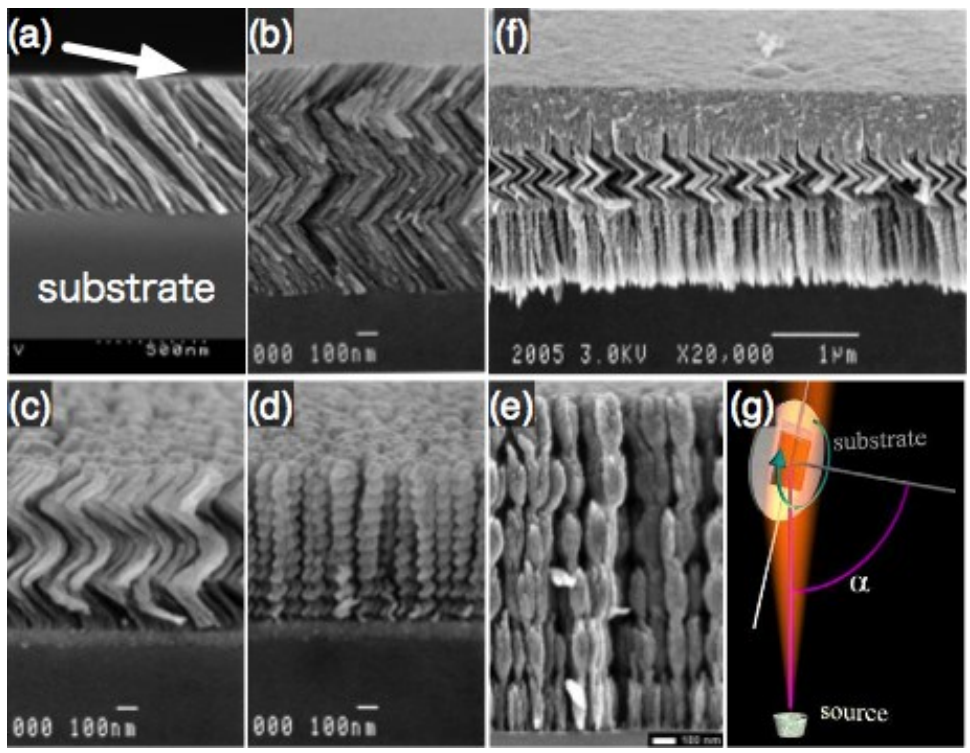

FIGURE 1. (a)-(f) Typical examples of the nanocolumnar structures of oblique-angle deposited (OAD) thin films, (g) a schematic drawing of the deposition geometry [17].
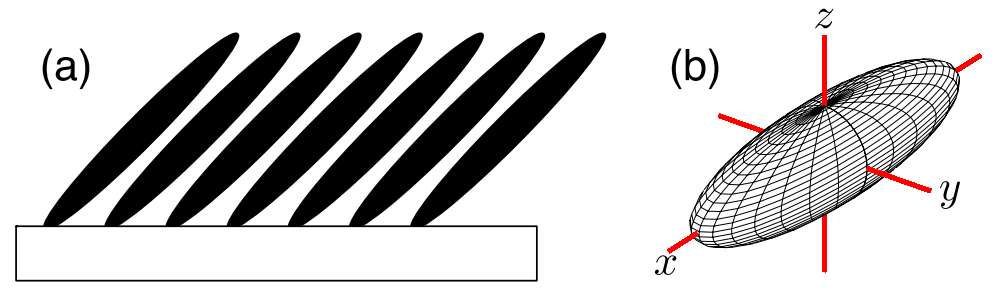

FIGURE 2. (a) Oblique columnar structure as an assembly of elongated spheroids, (b) dielectric spheroid. 


\section{PRACTICAL APPLICATIONS OF SHAPE-RELATED USEFUL PROPERTIES}

\section{Thin Film Waveplates [16, 18]}

Oblique columnar thin films of a transparent material show large birefringence ( $\Delta n \sim 0.5$ or larger) at a deposition angle of around $70^{\circ}$ [4]. This property is quite useful for application in thin film waveplates. Fig. 3 shows photographs of a $\mathrm{Ta}_{2} \mathrm{O}_{5}$ waveplate deposited in 1998 [18] and kept in ambient conditions in the author's office for more than 10 years. This film was deposited by using the Motohiro-Taga process [4] at a deposition angle of $70^{\circ}$ and has a chevron-shaped columnar cross section. As indicated in Fig. 3(a), the film is completely transparent. From the photograph taken through polarizers shown in Fig. 3(b), it is clear that the retardation properties are quite uniform. Therefore, the film is very durable and exhibits high uniformity over an area of $100 \mathrm{~cm}^{2}$. In addition, such a film is well reproducible because it was prepared by physical processes. Hodgkinson and $\mathrm{Wu}$ [19] succeeded in enhancing the birefringence by using a so-called serial bideposition technique, and very recently, Koike et al. [16] have developed practical thin film waveplates for optical pickups and liquid crystalline projectors.
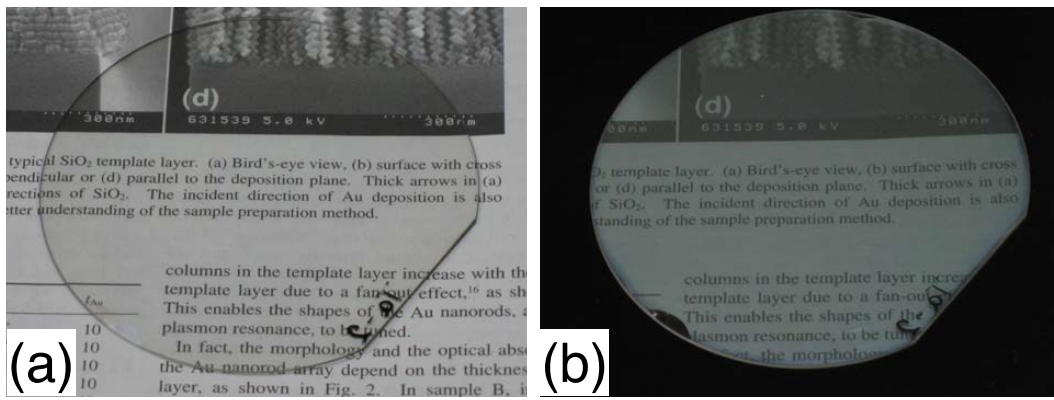

FIGURE 3. Visual appearance of a waveplate of an oblique-angle deposited (OAD) $\mathrm{Ta}_{2} \mathrm{O}_{5}$ thin film [18] taken (a) without and (b) with commercial sheet polarizers.

\section{Angular Selective Coatings [10]}

For the obliquely codeposited thin films with small metal particles are expected for the angular-selective coatings [10]. Oblique columnar thin films containing small metal particles show anisotropy in optical absorption depending on the polarization of the incident light and the angle of incidence. Small Ag particles can be embedded in the oblique columnar $\mathrm{SiO}_{2}$ by oblique codeposition [10]. The anisotropic transmittance properties for the obliquely codeposited $\mathrm{Ag}-\mathrm{SiO}_{2}$ are shown in Fig. 4(a). Measurements of the transmittance were performed in the plane of the vapor incidence as shown in Fig. 4(b). All spectra have absorption bands at wavelengths between 330 and $600 \mathrm{~nm}$. This large optical anisotropy in the obliquely codeposited $\mathrm{Ag}-\mathrm{SiO}_{2}$ thin films was explained in terms of the plasma resonance of $\mathrm{Ag}$ particles embedded in an anisotropic medium. Figures 4(c) and (d) are the visual appearances of $\mathrm{Ag}-\mathrm{SiO}_{2}$ thin films viewed from $\theta=+45^{\circ}$ and $\theta=-45^{\circ}$. They show clear, significant angular selectivity and may be useful for the coatings of inclined windows.

\section{Noble Metal Nanorod Arrays for SERS [14, 15]}

Much attention has been devoted to surface-enhanced Raman scattering (SERS) in the near-IR (NIR) region from the viewpoint of biochemical sensor applications. For application to biological materials, excitation in the NIR region is appropriate because this region is compatible with biological tissues' transparency window. Elongated nanoparticles (so-called nanorods) are strong candidates for NIR SERS substrates because the local field can be significantly enhanced at their ends. We have succeeded in aligning the nanorods end to end by using DOD [20]. Figure 5(a) shows the morphology of our Au nanorod arrays. Au nanorods were aligned on a $\mathrm{SiO}_{2}$ template layer (called the "shape control layer") having an anisotropic surface morphology prepared by oblique angle deposition (OAD) [15]. The shape control layer of $\mathrm{SiO}_{2}$ as the template for Au nanorods is prepared by a serial bideposition (SBD) technique. The surface of the shape control layer prepared by SBD is corrugated anisotropically. On the anisotropic shape control layer, Au is also evaporated obliquely in vacuum. The deposited $\mathrm{Au}$ is only around $10 \mathrm{~nm}$ thick on average. The Au sticks only to the top of the columns, owing to shadowing, and forms elongated 
nanoparticles (nanorods). Due to the in-line alignment of nanorods, the nanorod arrays show large dichroism in the NIR region corresponding to the local plasmon resonance, as shown in Fig. 5(b). Excellent SERS properties are observed, as indicated in Fig. 5(c), when Raman spectra are measured on Au nanorod arrays immersed in a solution of 4,4'-bipyridine. The SERS spectra can be detected down to $1 \mu \mathrm{M}$ of solution within a few minutes after the immersion of samples. In our measurement system, the spot size of the laser is around $1 \mu \mathrm{m}^{2}$. The thickness of the nanorod array is of the order of $10 \mathrm{~nm}$. Therefore, at $1 \mu \mathrm{M}$, the number of molecules existing inside the SERS active volume is estimated to be fewer than 10 molecules. Thus, our nanorod arrays are nearly sensitive enough to detect a single molecule. The high sensitivity can be attributed to the high number density of nanorods and effective field concentration between in-line aligned nanorods.

In addition to the high sensitivity, our nanorod arrays are well reproducible, and no contamination occurs during deposition. Moreover, they maintained high SERS sensitivity for more than one year. These are the advantages of the OAD technique. Fortunately, a Japanese coating company recently began to produce our nanorod arrays. These are already available on the market today.
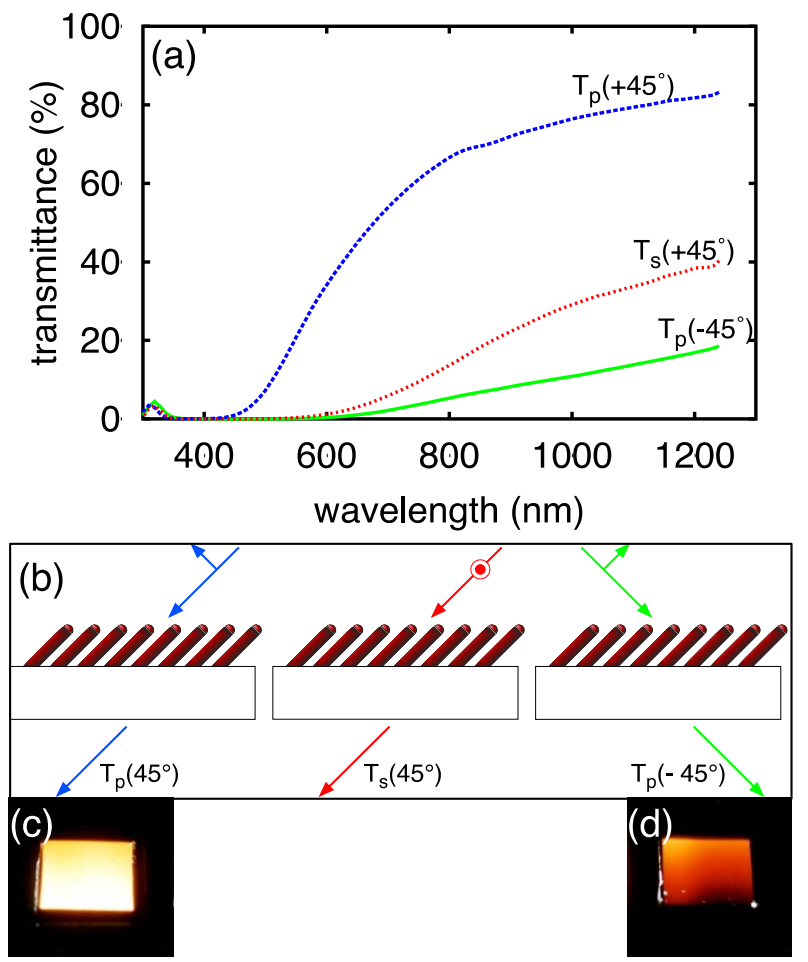

FIGURE 4. (a) Transmittance spectra of the $\mathrm{Ag}-\mathrm{SiO}_{2}$ angular selective coating, (b) definition of measurement geometry, (c) and (d) visual appearances at $+45^{\circ}$ and $-45^{\circ}$, respectively [10].
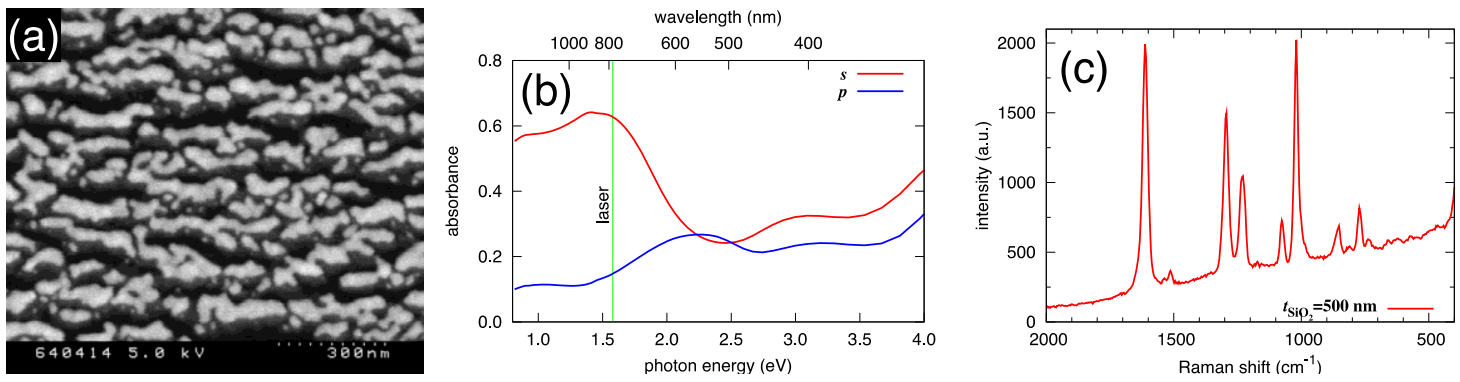

FIGURE 5. (a) Scanning electron microscopy image of the surface, (b) polarization-dependent absorbance spectra, and (c) surface-enhanced Raman scattering spectra of the Au nanorod array [15]. 


\section{Low-Reflectivity Wire-Grid Polarizers [11]}

Recent liquid-crystal (LC) projectors require high thermal durability for their optical elements. Metal wire-grid (WG) polarizers are quite suitable because they are not degraded by heat and light. In addition, high reflectivity of the wire-grid polarizers is useful for recycling the light near the light source. However, highly reflective polarizers downstream might generate stray light, which might degrade image quality. Thus, improving the brightness and durability of projectors requires the development of low-reflectivity (LR) WG polarizers.

From considerations based on optical admittance, we found that the reflectivity of conventional WG polarizers can be reduced if we fabricate the multilayered wires of absorptive and dielectric materials on highly reflective Al wires. At first, we prepare aluminum WG polarizers by interference lithography and dry etching. The dielectric layer of $\mathrm{SiO}_{2}$ is deposited by ordinary sputtering. The absorptive layer of $\mathrm{FeSi}_{2}$ is then deposited by ion beam sputtering at a glancing deposition angle of $87^{\circ}$ from the surface normal. Because of the shadowing effect, the sputtered $\mathrm{FeSi}_{2}$ adheres only to the top of the WGs covered with $\mathrm{SiO}_{2}$.

Figures 6(a) and (b) indicate the reflectance and transmittance spectra of a WG and an LR-WG polarizer. The reflectance of TE waves for the LR-WG polarizer is clearly much smaller than that for the WG polarizer. No significant degradation of the transmission properties is recognized. In Figs. 6(c) and (d), the visual appearances of these polarizers are displayed. These photos are taken through a commercial sheet polarizer of which the polarization axis is perpendicular (Fig. 6(c)) or parallel (Fig. 6(d)) to wires. The LR-WG polarizer clearly looks much darker than the WG polarizer. The durability of the LR-WG polarizers is basically identical to that of the conventional WG polarizers. Our LR-WG polarizers are now used in commercial LC projection displays that require high thermal durability.
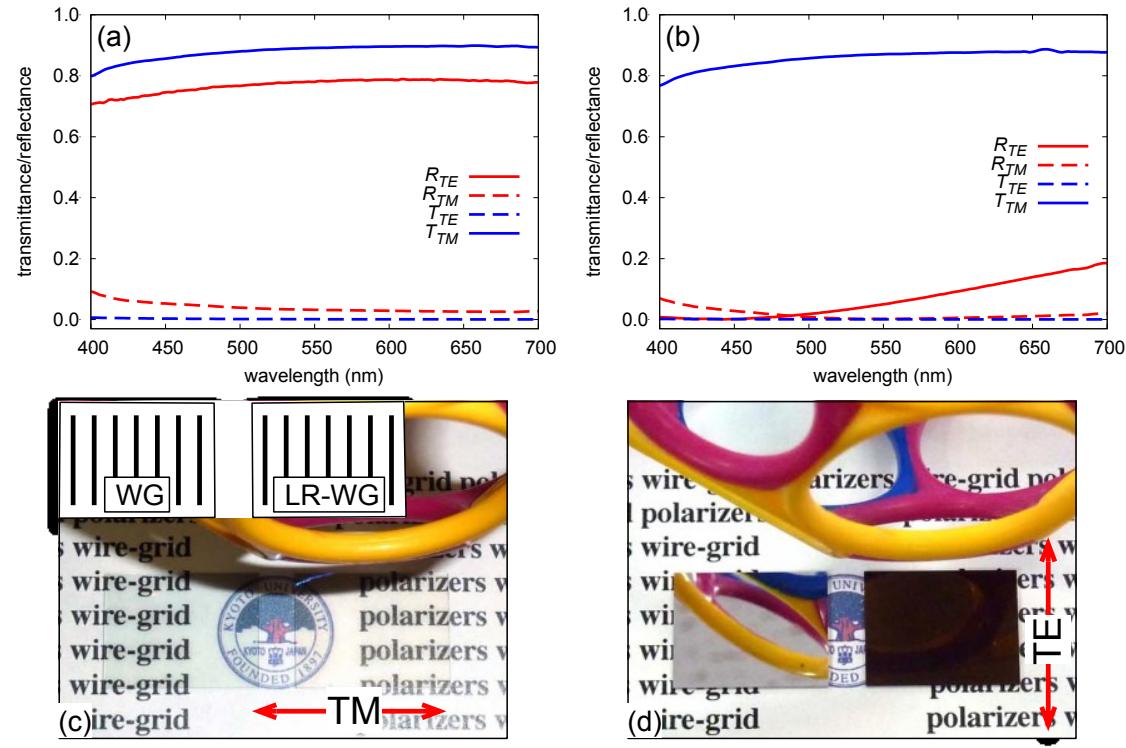

FIGURE 6. (a) and (b) Transmittance/reflectance spectra of wire-grid (WG) and low-reflectivity WG (LR-WG) polarizers, respectively. (c) and (d) Visual appearances of WG/LR-WG polarizers taken through a commercial sheet polarizer, the polarization axis of which is perpendicular (c) and parallel (d) to the wire grids [11].

\section{SUMMARY}

Unique nanocolumnar morphologies can be tailored by the DOD technique. Many of the shape-related useful properties of DOD thin films originate from the anisotropy in polarizability of elongated nanocolumns. In this article, examples of such shape-related properties have been reviewed. Applications include thin film waveplates, angular selective coatings, and noble metal nanorod arrays for SERS and LR-WG polarizers. Because tailoring nanomorphology by DOD is robust in selection of materials, more advanced applications are expected by integrating the functions of nanoshapes as well as functions of materials. 


\section{ACKNOWLEDGMENTS}

This article has been prepared in collaboration with all members of the Micro Process Engineering Laboratory at Kyoto University, Akio Takada and Nobuyuki Koike at Dexerials Corporation, and Yasunori Yaga at Chubu University, Japan. Student contributions were especially important for carrying out the investigations. I am also grateful to Sadamu Kinoshita of Kyoto University for the SEM observations. This work was supported by KAKENHI 25286037, 21656058, and 17310073.

\section{REFERENCES}

1. H.C. van de Hulst, Light Scattering by Small Particles, New York: Dover, 1981.

2. C.F. Bohren, D.R. Huffman, Absorption and Scattering of Light by Small Particles, New York: Wiley, 1983.

3. H.A. Macleod, J. Vac. Sci. Technol. A 4, 418 (1986).

4. T. Motohiro, Y. Taga, Appl. Opt. 28, 2466 (1989).

5. G.B. Smith, Opt. Commun. 71, 279 (1989).

6. K. Kuwahara, H. Hirota, Jpn. J. Appl. Phys. 13, 1093 (1974).

7. E.W. Pugh, J. Matisoo, D.E. Speliotis, E.L. Boyd, Journal of Applied Physics 31, S293 (1960).

8. R. Sugita, IEEE. Trans. Magn. MAG-20, 687 (1984).

9. R. Gontarz, Ratajcza.H, P. Suda, Physica Status Solidi 15, 137 (1966).

10. M. Suzuki, Y. Taga, J. Appl. Phys. 71, 2848 (1992).

11. M. Suzuki, A. Takada, T. Yamada, T. Hayasaka, K. Sasaki, E. Takahashi, S. Kumagai, Nanotechnology 21, 175604 (2010).

12. K. Robbie, M.J. Brett, A. Lakhtakia, J. Vac. Sci. Technol. A 13, 2991 (1995).

13. M. Suzuki, Y. Taga, Jpn. J. Appl. Phys. Part 2 40, L358 (2001).

14. M. Suzuki, W. Maekita, Y. Wada, K. Nakajima, K. Kimura, T. Fukuoka, Y. Mori, Appl. Phys. Lett. 88, 203121 (2006).

15. M. Suzuki, K. Nakajima, K. Kimura, T. Fukuoka, Y. Mori, Analytical Sciences 23, 829 (2007).

16. N. Koike, K. Sasaki, T. Yamada, N. Hanashima, A. Takada, M. Suzuki, Journal of Nanophotonics 8, 083991 (2014).

17. M. Suzuki, Journal of Nanophotonics 7, 073598 (2013).

18. M. Suzuki, T. Ito, Y. Taga, Proc. SPIE 3790 (1999) 94.

19. I. Hodgkinson, Q.H. Wu, Appl. Opt. 38, 3621 (1999).

20. M. Suzuki, W. Maekita, K. Kishimoto, S. Teramura, K. Nakajima, K. Kimura, Y. Taga, Jpn. J. Appl. Phys. Part 2 44, 1193 (2005) 